\title{
Rehabilitation of atrophic maxilla with tilted implants - Case report
}

\author{
Tavares $C^{1}$, Pires $C^{1}$ \\ Affiliations: \\ 1. SPI Research Centre, Porto \\ Portugal \\ Corresponding author: \\ Catarina Tavares \\ catarinaa1989@hotmail.com
}

\begin{abstract}
Purpose: An 81-year-old male patient, without systemic disorders, came to the appointment referring lack of masticatory function. It was planned a rehabilitation with six implants in upper atrophic maxilla (Implant Direct - Swish Plus), including immediate placing of 4 implants and 2 posterior tilted implants due to the severe pneumatization of maxillary sinus and bone ridge resorption. The prosthetic phase was initiated 4 months after implants surgery. Case report: The placement of tilted implants with the objective of the necessity of bone grafts and increase bone support has been reported by several authors as a viable rehabilitation. And since it is a minimally invasive surgery, it has good acceptance by the patient. The placement of tilted implants is a viable surgical alternative in anatomic regions such as: the anterior or posterior wall of the maxillary sinus, the palatal curvature or the pterygoid process. This treatment option, allied to the use of longer implants, allows an improved primary stability favoring immediate loading. Also, allows the adequate distribution of the implants, resulting in a more uniform distribution of forces and avoids the necessity of cantilever. Some authors have been questioning the biomechanical qualities of this surgical option; however, there are no statistical differences when compared with implants conventionally placed. Conclusions: Tilted implants allow an implant-supported rehabilitation of atrophic maxilla without bone grafts, which decreases the waiting period, the patient's morbidity and the costs of the treatment.
\end{abstract}

\section{KEYWORDS}

Oral rehabilitation, atrophic maxilla, tilted implants. 


\section{INTRODUCTION}

The rehabilitation of the edentulous maxilla has been discussed over the time through many treatment options with a high level of success and predictability. ${ }^{1-4}$ As soon as the centripetal alveolar resorption increases, implant placement becomes harder because of the decrease of bone quality, the pneumatization of maxillary sinus and the proximity of some anatomic structures like the nasopalatineal duct., 6

The placement of tilted implants with the objective of decreasing the necessity of bone grafts and increasing bone support has been reported by several authors and allows a viable rehabilitation, with minimally invasive surgery, that as good acceptance by the patient. ${ }^{7-9}$

The placement of tilted implants is a viable surgical alternative in anatomic regions such as the anterior or posterior wall of the maxillary sinus, the palatal curvature or the pterygoid process. ${ }^{10,11}$ This treatment option, allied to the use of longer implants, allows an increase of bone-implant contact (BIC) area and allows an improved primary stability favoring the immediate loading. It also, allows the adequate distribution of the implants, resulting in a more uniform distribution of forces and the decrease or elimination of the cantilever. $6-8,10,12$

This technique can be performed in patients with various systemic conditions which are often contraindications for bone grafting procedures. ${ }^{12}$

Besides these advantages, this technique requires special surgical skills and a learning curve. 5, 11,13 The use of 3D computer-guided implant planning programs and the use of a surgical template can be a very important tool to facilitate the surgical procedure and to carefully planned the implant inclination in order to avoid important anatomical structures. ${ }^{6,12}$ Some difficulties could be found in patients with limited mouth-opening and when implant emergence it's unfavorable for the prosthetic rehabilitation. ${ }^{11}$

\section{CASE REPORT}

81-year-old male patient, without systemic disorders appeared at the appointment referring lack of masticatory function. After the radiographic and clinic exam, we decided for an implant supported rehabilitation. It was planned the placement of 6 implants on the upper atrophic maxilla (Swish Plus Implant ${ }^{\mathrm{TM}}$-Implant Direct ${ }^{\circledR}$, USA), with immediate loading of 4 implants and 2 posterior tilted implants due to the severe pneumatization of maxillary sinus and bone ridge resorption. The prosthetic phases were

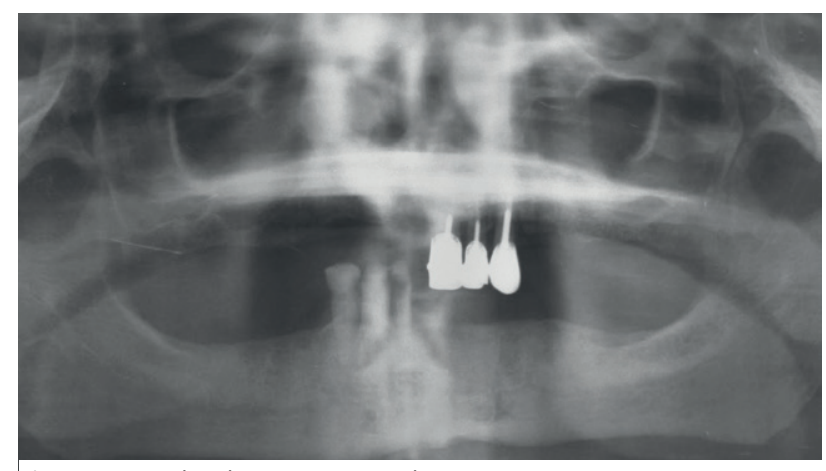

Figure 1. Initial orthopantomography

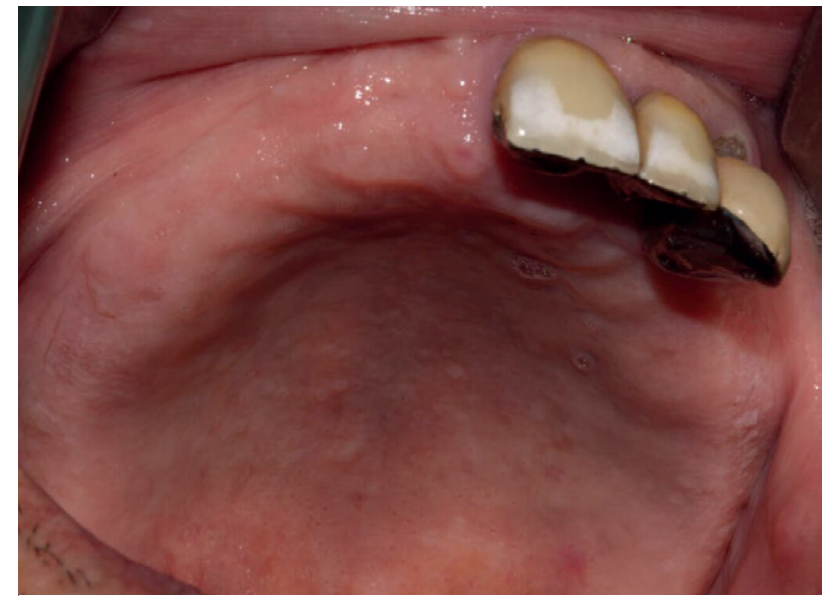

Figure 2. Initial photograph of of the patient

initiated 4 months after implant surgery. Patient was informed of the purpose of the treatment, as well as possible alternative treatments, and provided the written consent.

\section{Surgical technique}

Three days before surgery, and for each day for the first week afterward, patient rinsed twice daily with chlorhexidine digluconate 0.2\% mouthwash. Patient was premedicated with $875 \mathrm{mg}$ of amoxicillin and $125 \mathrm{mg}$ clavulanic acid, 3 days before surgery and twice a day for 1 week postoperatively. Glucocorticoid (prednisolone 20mg), and analgesics (paracetamol 1g) were prescribed. Surgery was performed under local anesthesia with articaine chorhydrate with adrenaline 1:100.000. Compromised teeth were extracted, and sockets were carefully debrided with sterile saline solution (Figure 2). 
The surgery was initiated by a mid-crestal incision in keratinized gingiva starting from first molar region to contralateral side and a mucoperiosteal flap was elevated exposing the vestibular bone wall. The posterior implants were tilted distally at about a 30-degree angle and placed parallel to the anterior wall of the maxillary sinus, with the following measures: $4.2 \times 13 \mathrm{~mm}$ and the 4 anterior implants $(4.2 \mathrm{~mm} \times 13 \mathrm{~mm})$ were placed in a vertical position (Figure 4, 5). Implants used were SwishPlus ${ }^{\mathrm{TM}}$ (Implant Direct $^{\circledR}$, USA). After component installation, a $30 \mathrm{~N} / \mathrm{cm}$ torque was applied following manufacturer's instructions. The flap was closed using monofilament non-absorbable polyamide surgical suture 4-0 (Polyamid, Medipac ${ }^{\mathrm{TM}}$, Greece) The spaces between the post-extrational alveolus and the implants were filled with autogenous bone particles removed from drill bits to low speed and xenogeneic bovine bone particles (DirectOss ${ }^{\mathrm{TM}}$ ) and bovine Collagen Matrix indicated for use in guided tissue (Reguarde ${ }^{\text {TM }}$ Resorbable Collagen Membrane) before closing the flap (Figure 6, 7). One week after surgery, sutures were removed, and patient was controlled monthly in follow-up appointments to both assess the soft tissue health and to adjust the provisional prosthesis (Figure 8).

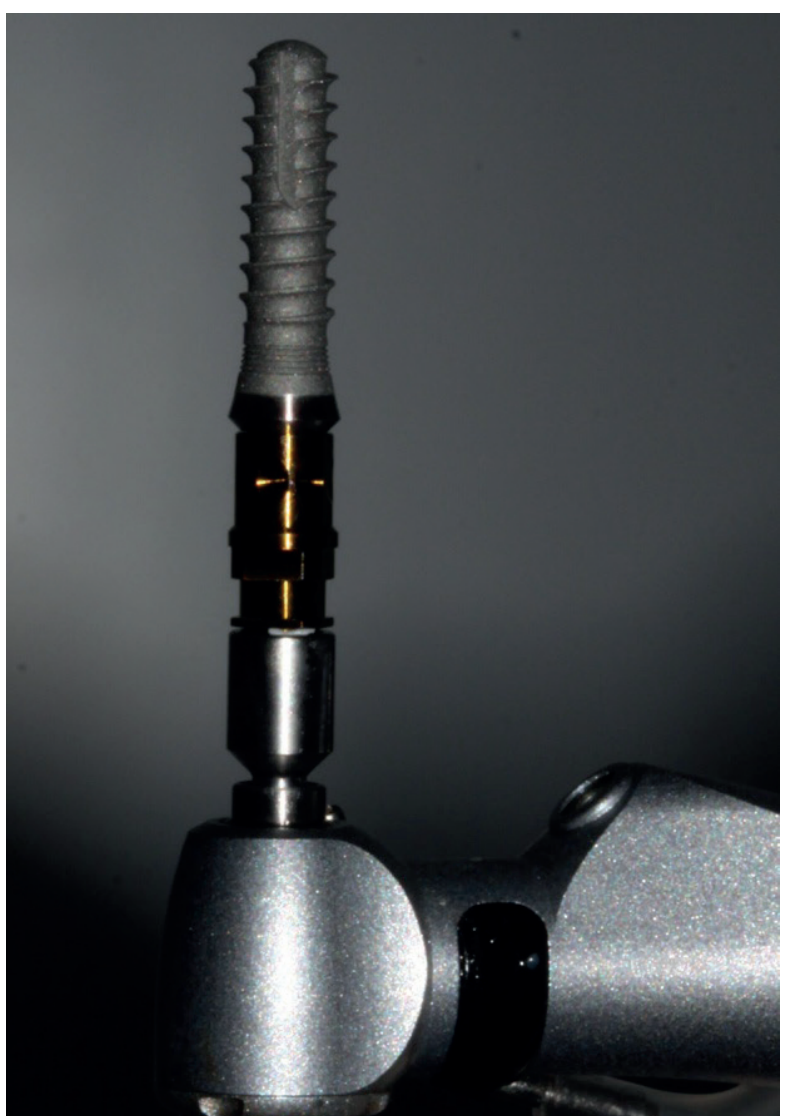

Figure 3. SwishPlus $13 \mathrm{~mm}$ Implant

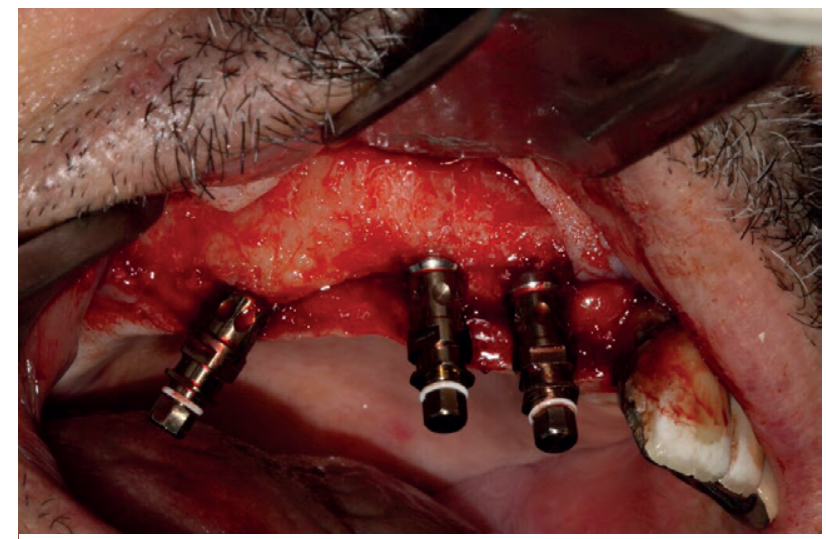

Figure 4. Implant placement at Upper Right Quadrant

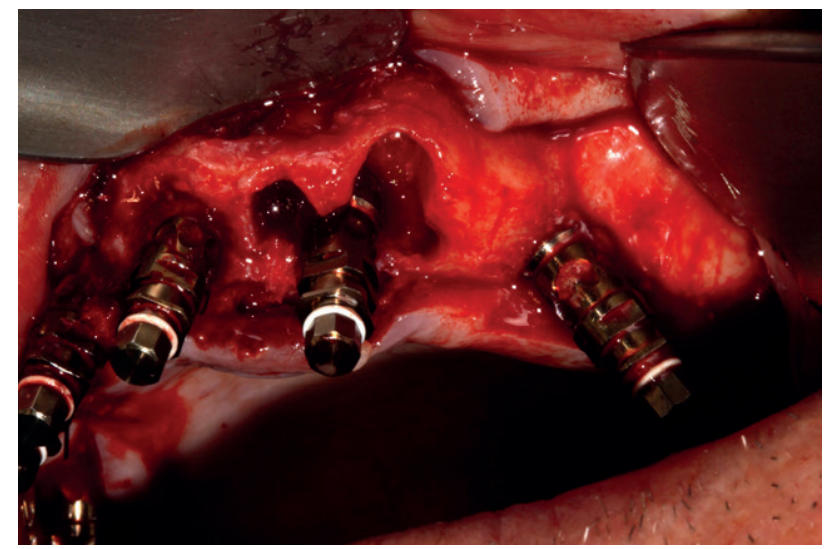

Figure 5. Implant placement at Upper Left Quadrant

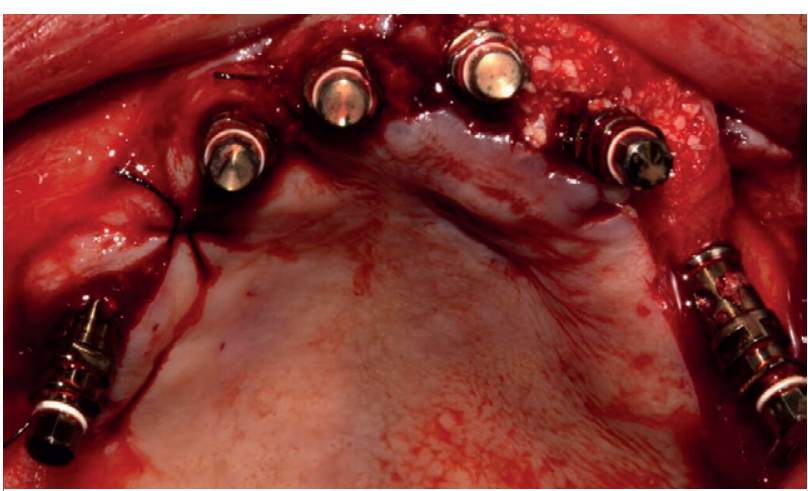

Figure 6. Post extraction filling of the sockets with autogenous bone

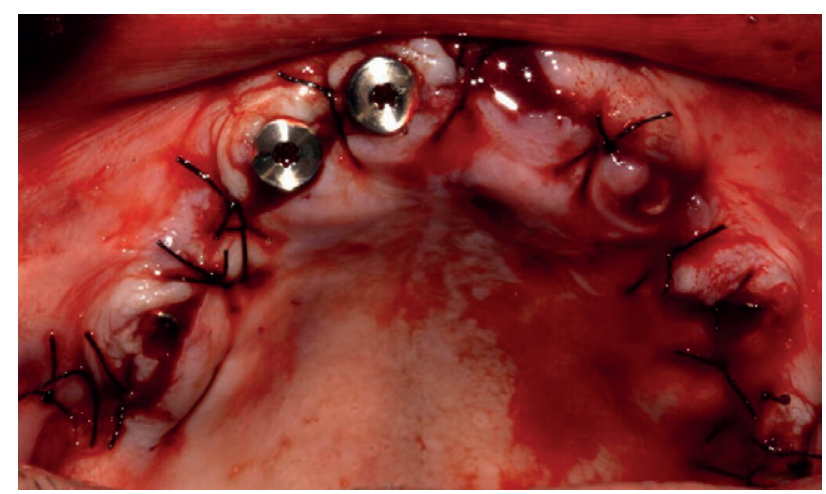

Figure 7. Suture 


\section{Prosthetic Design and Loading}

During the prosthetic phase, impressions and vertical dimension registration were taken. The impression pillars or transfers were linked with dental floss, and small increments of DuraLay ${ }^{\mathrm{TM}}$ (GO Pattern Resin, CO America Inc) (Figure 9). The impression was taken with vinylpolysiloxane material (addition silicone) (Hydrorise ${ }^{\mathrm{TM}}$, Zhermack ${ }^{\circledR}$, and Italy). Once the impression was taken, implant analogues were placed into the impression and all laboratory steps were followed in the conventional manner. The infrastructure was completed, radiographic and clinical settling were assessed.

Patient were rehabilitated with a hybrid prosthesis fixed by 6 implants on maxilla, and five implants on mandible (Figure 10). The healing time took about 6 months (Figure 8).

A final panoramic radiograph was taken to check implant position and the coupling between prosthetic components. The patient was given oral hygiene instructions and the screw access holes were covered with a provisional filling material. At the one-week evaluation visit, a definitive material was placed to close the screw access holes (Figure 11, 12).

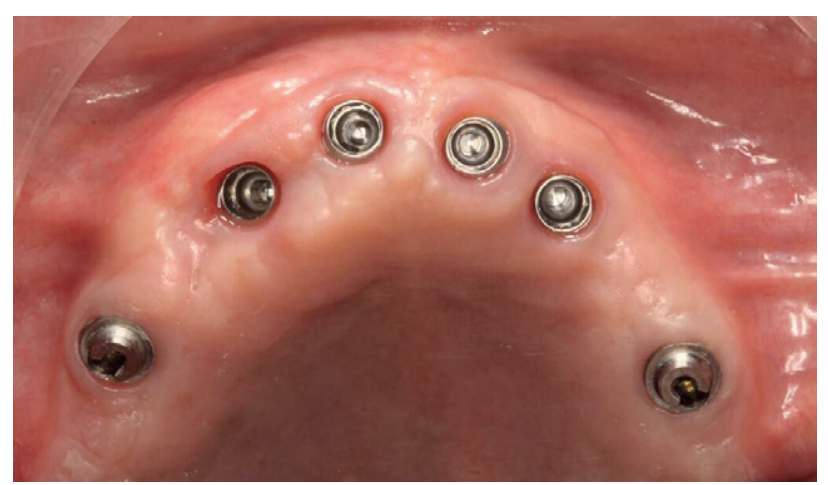

Figure 8. Post-healing image of gingival margin

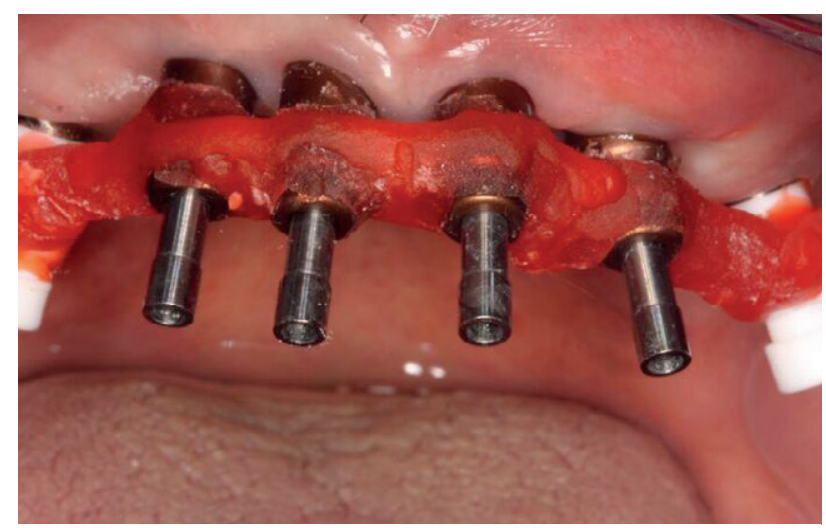

Figure 9. Splinting of impression pillars with duralay ${ }^{\mathrm{TM}}$

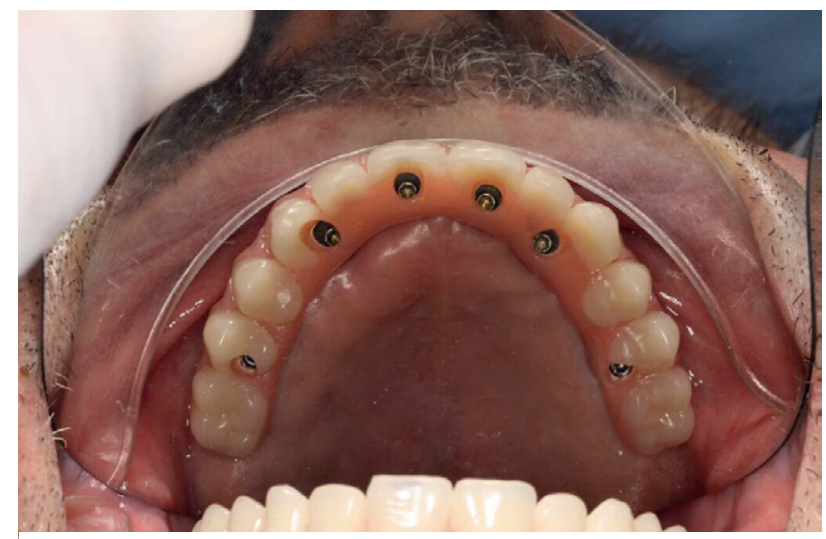

Figure 10. Hybrid overdenture prosthesis: occlusal view

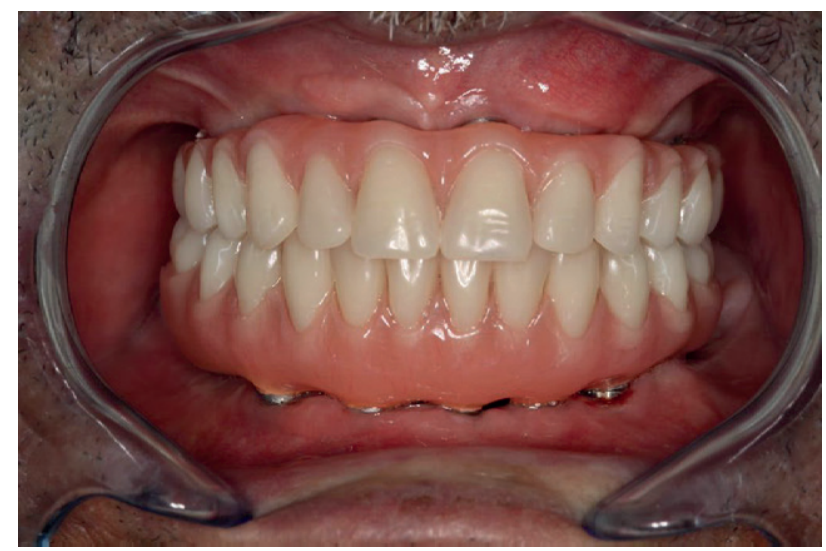

Figure 11. Final photograph

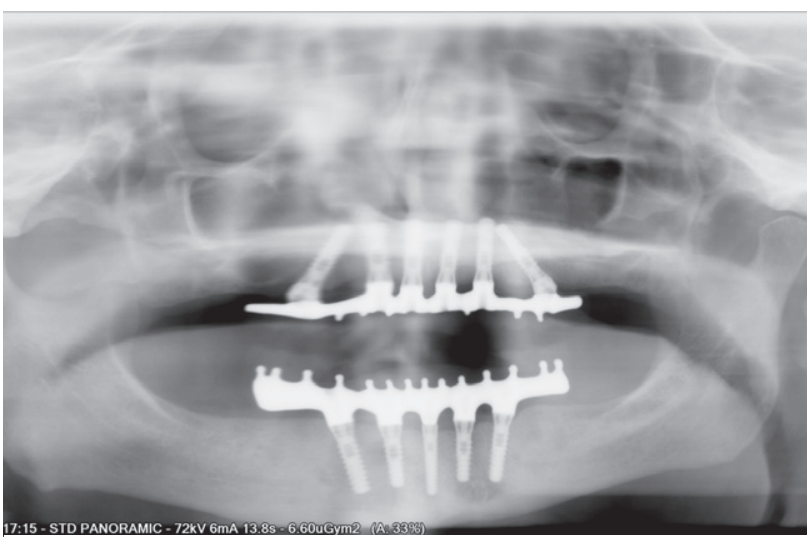

Figure 12. Final orthopantomography 


\section{DISCUSSION}

The main objective of this patient was to regain their masticatory function. It was a man with 81 years, that despite systemically healthy, preferred the least invasive possible treatment. We chose to put 6 implants in the upper arch (the distal were tilted), and 5 implants in the lower jaw. It was rehabilitated with two hybrid prosthesis. Tilted implants were $13 \mathrm{~mm}$, which enables to a higher initial primary stability.

The literature shows studies with high rates of success in applying tilted implants and reduced bone loss at short and medium term follow-ups.7.11 The treatment time is shorter and the costs are lower, which was also a decisive factor in the planning of this case. ${ }^{8}$

When we are planning our rehabilitation, we should evaluate the bone quality and quantity presented in the patient. The use of angulated implants should remain confined to situations of favorable bone quality. The inclination degree must be also evaluated. Greater inclinations of the implants $\left(>30^{\circ}\right)$ may increase force levels at the implant-bone and implant-abutment interfaces, so this should be avoided. ${ }^{12}$

Some studies report the possibility to occur biomechanics complications with this treatment option because of the marginal bone loss around tilted implants, however, there was not sufficient information to ground these speculations. ${ }^{4,7}$ Many authors defend the high success rate of this therapy when compared with conventional implant placement technique, and many other studies show this.8,10,11 Some authors refer that the most common prosthetic complication was the fracture of the acrylic prostheses, which occurred in $16.4 \%$ and $19 \%$ of the cases respectively..$^{3,13}$ Other author only found screw loosening, which occurred in $17.5 \%$ of the provisional prostheses. ${ }^{8}$

Studies of rehabilitated 20 maxilla's using 6 implants, 2 anterior axial implants and 4 tilted implants, 2 parallel to the anterior sinus wall and 2 parallel to the posterior wall, showed that, after 31.3 months average follow-up, 97.5\% of the axial implants and $99.2 \%$ of the tilted implants survived. ${ }^{3}$ Other authors have reported high success rates with both delayed and immediately loaded full-arch prosthesis. 8,13

The prosthetics complications with hybrid prosthesis are frequent and the acrylic veneer fracture is the most common. Some disadvantages are related to the expensive maintenance of that prosthesis. The surgeon needs to be very trained in this type of technique. ${ }^{12}$

The level of evidence of the included studies was rather poor because no randomized clinical trials neither comparative prospective trials were found. We mostly found prospective single cohort or multicenter studies witch were at high risk of bias.

We need more research with more extensive follow-up times to assess whether this stability and success rate remains constant over time.

\section{CONCLUSIONS}

Tilted implants allow an implant-supported rehabilitation of atrophic maxilla, decreasing the waiting time, the patient's morbidity and the costs of the treatment, being a predictable treatment with good results and a high level of success and patient satisfaction. Tilted implants are an effective and safe alternative to maxillary sinus floor augmentation procedures and allow for longer implants to be placed with improved bone anchorage.4, 8,11,12

\section{CONFLICT OF INTEREST}

The authors declares that there is no conflict of interest regarding the publication of this article. 


\section{REFERENCES}

1. Calandriello R, Tomatis M. Simplified treatment of the atrophic posterior maxilla via immediate/early function and tilted implants: A prospective 1-year clinical study. Clinical implant dentistry and related research. 2005;7 Suppl 1:S1-12.

2. Penarrocha-Oltra D, Candel-Marti E, Ata-Ali J, Penarrocha-Diago M. Rehabilitation of the atrophic maxilla with tilted implants: review of the literature. The Journal of oral implantology. 2013;39(5):625-32.

3. Agliardi E, Clerico M, Ciancio P, Massironi D. Immediate loading of full-arch fixed prostheses supported by axial and tilted implants for the treatment of edentulous atrophic mandibles. Quintessence international. 2010;41(4):285-93.

4. Ata-Ali J, Penarrocha-Oltra D, Candel-Marti E, Penarrocha-Diago M. Oral rehabilitation with tilted dental implants: a metaanalysis. Medicina oral, patologia oral y cirugia bucal. 2012;17(4):e582-7.

5. Del Fabbro M, Bellini CM, Romeo D, Francetti L. Tilted implants for the rehabilitation of edentulous jaws: a systematic review. Clinical implant dentistry and related research. 2012;14(4):612-21.

6. Del Fabbro M, Ceresoli V. The fate of marginal bone around axial vs. tilted implants: a systematic review. European journal of oral implantology. 2014;7 Suppl 2:S171-89.

7. Monje A, Chan HL, Suarez F, Galindo-Moreno P, Wang HL. Marginal bone loss around tilted implants in comparison to straight implants: a meta-analysis. The International journal of oral \& maxillofacial implants. 2012;27(6):1576-83.

8. Testori T, Del Fabbro M, Capelli M, Zuffetti F, Francetti L, Weinstein RL. Immediate occlusal loading and tilted implants for the rehabilitation of the atrophic edentulous maxilla: 1-year interim results of a multicenter prospective study. Clinical oral implants research. 2008;19(3):227-32.

9. Chrcanovic BR, Albrektsson T, Wennerberg A. Tilted versus axially placed dental implants: a meta-analysis. Journal of dentistry. 2015;43(2):149-70.

10. Pozzi A, Sannino G, Barlattani A. Minimally invasive treatment of the atrophic posterior maxilla: a proof-of-concept prospective study with a follow-up of between 36 and 54 months. The Journal of prosthetic dentistry. 2012;108(5):286-97.

11. Menini M, Signori A, Tealdo T, Bevilacqua M, Pera F, Ravera G, et al. Tilted implants in the immediate loading rehabilitation of the maxilla: a systematic review. Journal of dental research. 2012;91(9):821-7.

12. Asawa N, Bulbule N, Kakade D, Shah R. Angulated implants: an alternative to bone augmentation and sinus lift procedure: systematic review. Journal of clinical and diagnostic research : JCDR. 2015;9(3):ZE10-3.

13. Francetti L, Rodolfi A, Barbaro B, Taschieri S, Cavalli N, Corbella S. Implant success rates in full-arch rehabilitations supported by upright and tilted implants: a retrospective investigation with up to five years of follow-up. Journal of periodontal \& implant science. 2015;45(6):210-5. 